\title{
Decoupled Control of Carbon Nanotube Forest Density and Diameter by Continuous-Feed Convective Assembly of Catalyst Particles
}

\author{
Erik S. Polsen, Mostafa Bedewy, and A. John Hart*
}

\begin{abstract}
The widespread potential application of vertically aligned carbon nanotube (CNT) forests have stimulated recent work on large-area chemical vapor deposition growth methods, but improved control of the catalyst particles is needed to overcome limitations to the monodispersity and packing density of the CNTs. In particular, traditional thin-film deposition methods are not ideal due to their vacuum requirements, and due to limitations in particle uniformity and density imposed by the thin-film dewetting process. Here, a continuous-feed convective self-assembly process for manufacturing uniform mono- and multi-layers of catalyst particles for CNT growth is presented. Particles are deposited from a solution of commercially available iron oxide nanoparticles, by pinning the meniscus between a blade edge and the substrate. The substrate is translated at constant velocity under the blade so the meniscus and contact angle remain fixed as the particles are deposited on the substrate. Based on design of the particle solution and tuning of the assembly parameters, a priori control of CNT diameter and packing density is demonstrated. Quantitative relationships are established between the catalyst size and density, and the CNT morphology and density. The roll-to-roll compatibility of this method, along with initial results achieved on copper foils, suggest promise for scale-up of CNT forest manufacturing at commercially relevant throughput.
\end{abstract}

\section{Introduction}

Owing to the growing interest in developing products that use organized assemblies of carbon nanotubes (CNTs), high throughput and cost-effective manufacturing methods are required. Continuous processes are needed to scale-up the production of CNT forests to large-area applications,

E. S. Polsen, M. Bedewy, Prof. A. J. Hart Mechanosynthesis Group

Department of Mechanical Engineering University of Michigan

2350 Hayward St. Ann Arbor, MI 48109, USA

E-mail: ajohnh@umich.edu

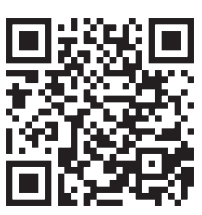

DOI: $10.1002 /$ smll.201202878 such as filtration membranes, dry adhesives, and thermal and mechanical interface layers. ${ }^{[1-5]}$ Moreover, in order to engineer the performance of CNTs in these applications, it is necessary to control the diameter and packing density of the CNTs within the forest. ${ }^{[5-9]}$ Nevertheless, both fundamental and practical understanding of CNT forest growth by chemical vapor deposition (CVD) remains largely limited to empirically derived recipes that are used in batch processes, such as on silicon wafers or pieces of wafers. As a result, the packing density of CNT forests is typically no more than a few percent relative to an ideal CNT solid, and the diameter, alignment, and spacing of individual CNTs within the forest are not uniform. ${ }^{[10-12]}$

The size and organization of the catalyst particles are critical to determining the characteristics of the resultant CNT forest. It is well known that the catalyst particle size dictates 
the CNT diameter. ${ }^{[13]}$ Moreover, because each particle can give rise to one CNT, the density of the particles, and their activation according to the growth conditions, determines the CNT forest density. The most prevalent method of catalyst preparation for substrate-bound CVD growth of CNTs is by annealing thin films deposited by electron beam evaporation or sputtering. Upon heating in a controlled atmosphere, the internal stresses and defects in the thin film catalyst cause it to form particles; this general process is known as thin-film dewetting. ${ }^{14]}$ Although the average size (diameter) of the particles can be controlled by the film thickness and the dewetting conditions (e.g., duration, temperature, atmosphere), dewetting of a thin film always results in a wide distribution of sizes that continue to evolve during subsequent annealing and even during CNT growth. ${ }^{[12,15-17]}$ Due to this polydispersity of catalyst particle sizes, CNTs grown by this method typically exhibit a wide distribution of diameters and resultant properties. In addition, this variance in particle diameter leads to a distribution of particle activation behavior. ${ }^{[18]}$ Typically, only $1 \%$ to $10 \%$ of all of the particles on the substrate yield CNTs, and this may be governed in part by the size distribution of the catalyst particles. ${ }^{[19]}$

Moreover, the achievable areal density of particles is inherently limited by the thin-film dewetting process and diffusion of the catalyst into the substrate. Recently, Esconjauregui and colleagues showed that CNT forests with record high packing density can be made by iterating sputtering and annealing operations to achieve high-density catalyst arrays. $^{[11]}$ A bulk density equal to $62 \%$ of a closely packed array was reported for double-wall CNTs with $2.4 \mathrm{~nm}$ average outer diameter. However, because of the need for vacuum deposition equipment and iterative processing, it is desirable to explore alternative methods to achieve high-density catalyst arrays, which leverage scalable wet chemistry synthesis of nanoparticles.

Methods for continuous solution-based coating of thin films are widespread in industry, including gravure and slotdie coating (also called zone- or tape-casting). ${ }^{[20,21]}$ These are easily implemented by spreading a thin film of liquid onto a moving web such as a metal foil. Convective selfassembly, which is analogous to slot-die coating, can be used to assemble organized arrays of micro- and nanoparticles on substrates in a continuous-feed manner. In this process, which is sometimes called 'doctor blade coating' or 'blade casting,' particles are spread by convection as the blade is translated across the surface of the substrate (or vice-versa). Depending on the balance between the evaporation rate at the meniscus and rate of meniscus translation across the substrate, monolayers or multilayers of particles can be achieved. ${ }^{[22-26]}$ Blade casting has been used to assemble both microparticles ${ }^{[22-24,26]}$ and nanoparticles ${ }^{[25]}$ over macroscopic areas. This evaporative self-assembly process is scalable and potentially costeffective compared to vacuum deposition methods because an indefinite substrate as a flexible foil or film can be used, because the process can occur at ambient conditions, and because $100 \%$ of the particles end up in the final assembly.

However, most previous research on convective assembly has focused on assembly of relatively large particles, such as $0.1-1 \mu \mathrm{m}$ diameter polymer particles for photonic applications, compared to the small sizes and different materials (e.g., selected transition metals such as Fe) that are suitable as catalysts for CNT growth. Additionally, while the goal of convective assembly is often to create close-packed monolayers, for CNT forest engineering it would be desirable to control the packing fraction by understanding of the governing assembly mechanisms and exploration of the parameter space.

Monodisperse Fe oxide nanoparticles can be made by several wet chemistry methods, ${ }^{[27-31]}$ and incidentally $\mathrm{Fe}$ oxide nanoparticle solutions are the basis of 'ferrofluids' which have many commercial applications including liquid seals for rotating shafts, contrast agents for magnetic resonance imaging (MRI), damping and heat transfer fluids for audio speakers, magnetofluid lubricants, and active suspension systems. Due to their broad commercial applications, ferrofluids are available in large quantities at low cost. For this reason, we chose to investigate blade-casting of ferrofluids as a lowcost means of catalyst array manufacturing for large-area CNT film growth. In previous work, CNT films and forests have been grown from pre-made nanoparticles deposited on substrates, including particles from ferrofluids; ${ }^{[32-34]}$ however, the typical method of spin-coating to deposit the particles is not readily scalable to continuous-feed processing, ${ }^{[35,36]}$ which is needed to develop roll-to-roll manufacturing of CNT forests on flexible substrates.

In this paper, we show that convective assembly enables nanoparticle array manufacturing with decoupled control of particle size and packing fraction, therefore enabling broadrange tuning of the diameter and packing density of CNT forests. Uniform CNT arrays with CNT diameters of 10 to $25 \mathrm{~nm}$, are demonstrated by our method, at continuous deposition speeds up to $0.4 \mathrm{~mm} \mathrm{~s}^{-1}$. Comparing quantitative AFM image analysis to CNT forest density, we calculate the catalyst yield for samples with monolayer catalyst particle deposits to be at or below $6 \%$. While the catalyst activation is low, we achieve CNT forest densities that are not only controllable, independent of catalyst particle diameter, but are 3-fold higher than achieved using a standard single deposition of thin film catalyst. Additionally, we present the successful growth of VACNT arrays on $\mathrm{Cu}$ foils with our method. Our results indicate the potential to significantly increase CNT forest density and yield, and to immediately enable roll-toroll manufacturing of CNT forests for emerging commercial applications.

\section{Results and Discussion}

\subsection{Governing Principles}

Assembly of pre-made nanoparticles into an organized array potentially allows decoupled control of the particle diameter and packing fraction. Drawing from previous studies of microparticle assembly by blade-casting, a necessary condition is for the supply of particles to the moving meniscus to match the deposition rate of particles into the array at the liquid interface. Hence, the required volume of particle solution needed to achieve a monolayer of particles having a 


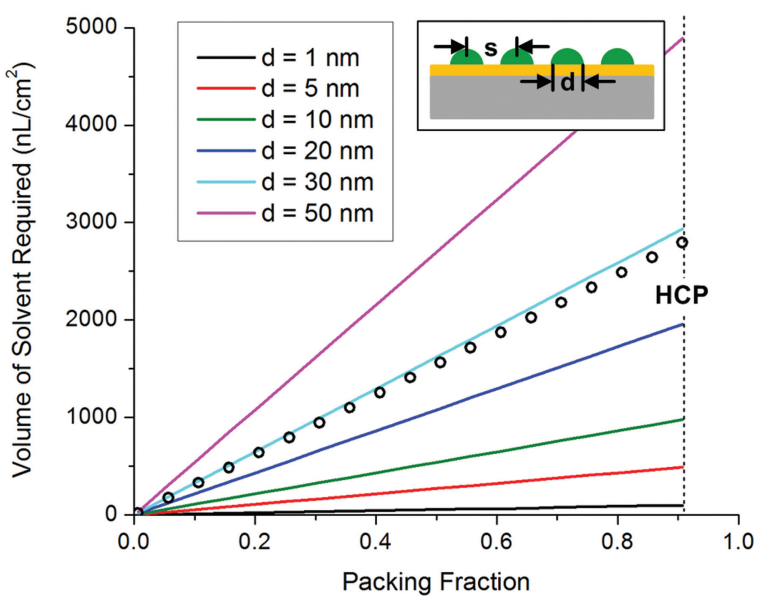

Figure 1. Volume of solvent (3.20 $\mathrm{mg} \mathrm{mL}^{-1}$ particle mass concentration) required for convective assembly of a particle monolayer of desired packing fraction, where individual data points represent the ferrofluid (MSGW11, FerroTec) used in this study.

certain packing fraction can be calculated a priori, as shown in Figure 1.

As the moving blade draws the solution across the substrate, the stretched meniscus slips over the substrate surface (Figure 2). Evaporation of the solvent causes convective flow of particles toward the meniscus. The particles in this region may assemble into well-ordered arrays by two complementary mechanisms, depending on the particle size and surface chemistry, the liquid surface tension, and other process parameters. ${ }^{[23,37-40]}$ For polymer microparticles, Dimitrov et al. first discussed how particles from the bulk solution are drawn into the meniscus via convective flow due to the evaporation of the solvent, then lateral capillary forces draw the particles together into an ordered configuration as the interstitial solvent evaporated. ${ }^{[40]}$ Studying dodecanethiol-ligated gold $(\mathrm{Au})$ nanoparticles dispersed in toluene Bigioni et al. showed that the particles are drawn to the air-liquid interface due to convective flow. Ordered islands of particles formed at the interface and were eventually pinned to the substrate as the meniscus receded along the three-phase contact line. ${ }^{[39]}$ During this pinning process, lateral capillary forces drew the islands into contact.

Because water-based nanoparticle solutions in our present work have a relatively low evaporation rate (of solvent), we believe the convective assembly model first proposed by Dimitrov is appropriate for this analysis. According to Dimitrov and Nagayama, the linear growth rate $\left(v_{c}\right)$ of a particle monolayer or multilayer, undergoing convective assembly is equal to: ${ }^{[40]}$

$v_{c}=\frac{\beta l j_{e} \varphi}{h(1-\varepsilon)(1-\varphi)}$

where $\beta$ is a coefficient of proportionality, $j_{\mathrm{e}}$ is the volumetric evaporation flux per unit area of the solvent, $l$ is the evaporation length (see Supporting Information), $\phi$ is the particle volume fraction in the suspension, $h$ is the thickness of the layer, and $\varepsilon$ is the porosity of the array. In order to maintain
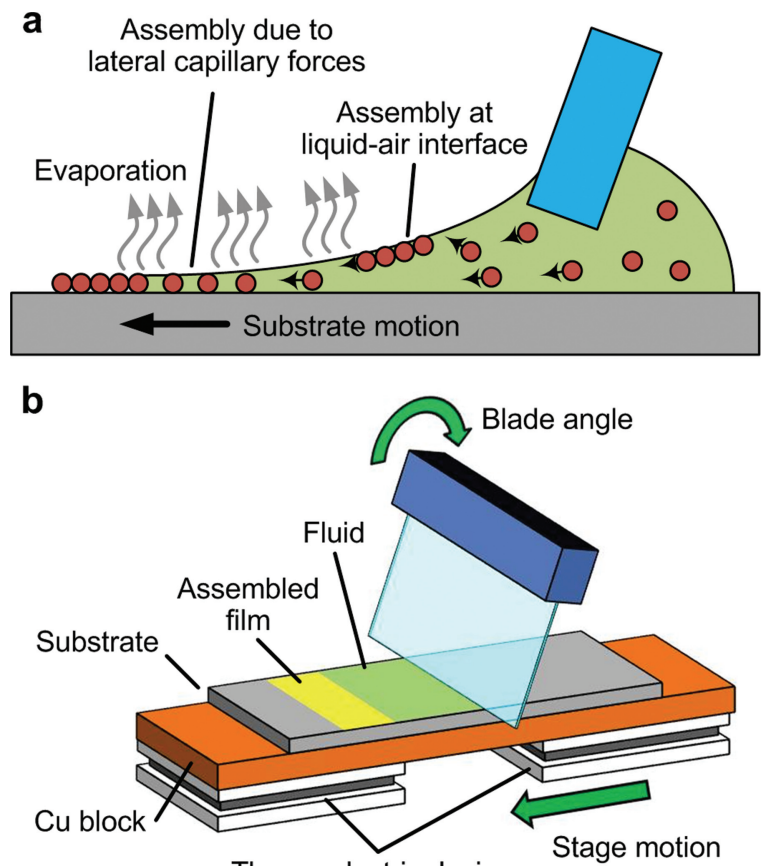

Thermoelectric devices
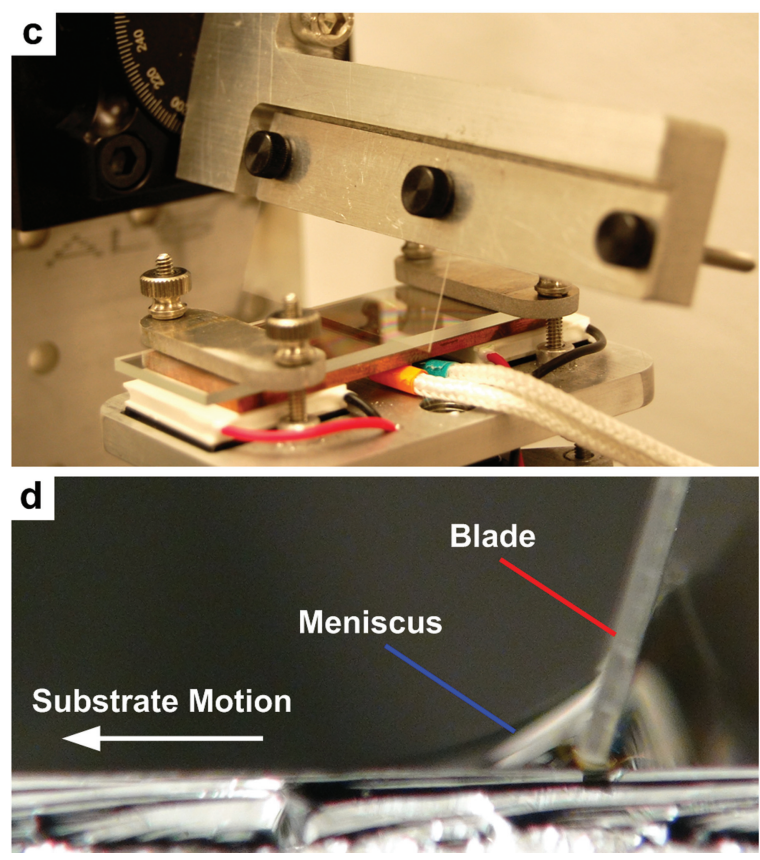

Figure 2. Nanoparticle array manufacturing by blade-casting. (a) Schematic of blade-casting process and mechanism of particle assembly at the liquid interface. (b) Schematic and (c) photograph of setup used for blade-casting, where silicon wafer substrate is placed on a copper platform supported by two thermoelectric chips. (d) Photograph during blade-casting of $27.20 \mathrm{mg} \mathrm{mL}^{-1}$ ferrofluid at $25 \mu \mathrm{m} \mathrm{s}^{-1}$. Although this apparatus has temperature control capability, all experiments in this study were performed at room temperature.

a steady growth rate of a particle monolayer according to Equation 1, the relative motion speed between the blade and the substrate $\left(v_{r}\right)$ must equal $v_{c}$. If $v_{r}>v_{c}$, a non-close-packed particle layer will be formed, whereas if $v_{r}<v_{c}$, multiple particle layers will be formed. This model is later used to interpret our findings of how the blade-casting process parameters 
for nanoparticles are related to the array morphology, and to the resultant CNT films grown from these particles by CVD. Moreover, this model can be used to determine the volume of solution (Figure 1) needed to form a monolayer of nanoparticles on the substrate, in contrast to the coupling between film thickness and particle size that is observed for thin film dewetting.

\subsection{Nanoparticle Film Assembly by Blade-Casting}

We first investigated the convective assembly of iron oxide (magnetite, $\mathrm{Fe}_{3} \mathrm{O}_{4}$ ) nanoparticles, which were obtained commercially as a water-based dispersion (ferrofluid MSGW11, FerroTec). The average particle diameter of this dispersion, as measured by dynamic light scattering (DLS), was $28.6 \pm$ $11.2 \mathrm{~nm}$ (Supporting Information, Figure S1). The particle concentration of the fluid was controlled by diluting the asreceived fluid with deionized water.

Using the custom-built apparatus shown in Figure 2c, solutions with particle concentrations varying from 0.33 to $163.2 \mathrm{mg} \mathrm{mL}^{-1}$ were blade-casted onto $15 \times 25 \mathrm{~mm}$ silicon wafer pieces. The substrates had been previously coated with $10 \mathrm{~nm} \mathrm{Al}_{2} \mathrm{O}_{3}$ by sputtering (see Methods). A droplet of fluid was applied at the interface between the blade (angled at $20^{\circ}$ from the vertical axis) and the substrate using a handheld pipette, and the substrate motion was started at a constant speed. For each experiment, the substrate speed was maintained at a constant value between $10 \mu \mathrm{m} \mathrm{s}^{-1}$ and $500 \mu \mathrm{m} \mathrm{s}^{-1}$. Once reaching steady-state, the dynamic contact angle between the meniscus and the substrate did not change considerably because a sufficient solution volume was used such that the evaporation of the solvent during the process did not cause a change to the contact angle. After the blade reached the end of the substrate, the remaining fluid was removed from the substrate. Any extra fluid must be removed quickly, before depinning of the meniscus from the blade, in order to prevent a backflow of the fluid onto the deposited area.

We found that the blade-casting parameters and substrate surface energy significantly influenced the density and uniformity of the assembled nanoparticle layers. Figure 3 shows AFM images of nanoparticle layers obtained at speeds ranging from $10-50 \mu \mathrm{m} \mathrm{s}^{-1}$, with and without oxygen plasma treatment of the substrate prior to blade casting. As expected from the governing principles of convective assembly, the coverage of the substrate with particles decreases with increasing deposition speed. Below the speed at which a uniform monolayer is observed, multilayer particle arrays are formed. Above this speed, reductions in the monolayer packing fraction are observed, and eventually we observe intermittent areas of particles on the substrate. For example, this is seen by comparing the results at $v_{r}=10 \mu \mathrm{m} \mathrm{s}^{-1}$ (multilayer) to $v_{r}=$ $50 \mu \mathrm{m} \mathrm{s}^{-1}$ (intermittent). We also observed that the particle coverage is proportional to the particle concentration in the starting solution (Supporting Information, Figure S2).

On substrates that were not plasma treated prior to bladecasting, we observed non-uniform multi-layer aggregates of particles rather than uniform monolayers. We attribute this difference to the influence of plasma treatment on the wetting of the substrate; as shown in Figure 3b,c, plasma treatment results in a significantly lower contact angle between the fluid and the substrate. The large contact angles measured on the non-treated substrates confirm that the interaction between the solution and the $\mathrm{Al}_{2} \mathrm{O}_{3}$ was too weak to enable meniscus pinning during blade casting, and therefore the iron oxide nanoparticles were not uniformly deposited on the substrate when pinning does not occur.

Further, on non-treated substrates, we observed that at the lower particle concentrations, or at high velocities (exceeding those shown in Figure 3), the ferrofluid did not sufficiently wet the substrate. Because of this, the meniscus slid across the substrate surface and did not pin. This resulted in samples where no visible film of particles was present after the deposition. Malaquin et al. previously studied convective assembly of polymer microparticles, and observed that where above a critical contact angle, typically $>20^{\circ}$, the particles were no longer pinned to the substrate by the meniscus and were instead carried away by the evaporation front. ${ }^{[23]}$ However, we observed that at the higher particle concentrations, the meniscus did form a lower contact angle, but at increased velocities the pinning to the substrate was not sufficiently strong to prevent a stick-slip motion of the ferrofluid as it moved across the substrate with the blade. This motion of the meniscus resulted in visible striations on the substrate surface parallel to the blade edge once the deposition was complete. Conversely, the fluid on the plasma-treated substrates always exhibited a low contact angle meniscus that had a stable, pinned evaporation front, and generated uniform particle films on the substrates. Samples processed within this range of the parameter space of concentrations and blade velocities had a uniform color (Supporting Information, Figure S3); this was a straightforward means of screening the results.

Additionally, on non-treated substrates at high velocity, a negligible coverage of particles was deposited because the contact angle is high and the evaporation front moves too rapidly to allow particles to deposit on the surface. However, because the contact angle between the ferrofluid and the plasma-treated substrates was significantly lower than that of non-treated substrates, particles were deposited under a broader range of solution concentrations and substrate velocities. Also, the static contact angle of the fluid was found to decrease with particle concentration on the non-treated substrates (Figure 3c), which was expected due to the direct coupling between particle concentration and surfactant concentration. However, the opposite trend was observed on plasma-treated substrates (Figure 3b), and this is most likely explained by the development of charged surface groups on the substrates due to the plasma treatment, and their interaction with similarly charged particles in the ferrofluid (zeta potentials from -30 to $-50 \mathrm{mV}$ ).

\subsection{Control of CNT Morphology and Density}

After blade-casting, all nanoparticle-coated substrates were exposed to the same CVD recipe, involving a reduction step $\left(775{ }^{\circ} \mathrm{C}, 100 / 400 \mathrm{sccm} \mathrm{He} / \mathrm{H}_{2}\right)$, followed a hydrocarbon exposure step $\left(775{ }^{\circ} \mathrm{C}, 400 / 100 / 100 \mathrm{sccm} \mathrm{He} / \mathrm{H}_{2} / \mathrm{C}_{2} \mathrm{H}_{4}\right)$. We found 

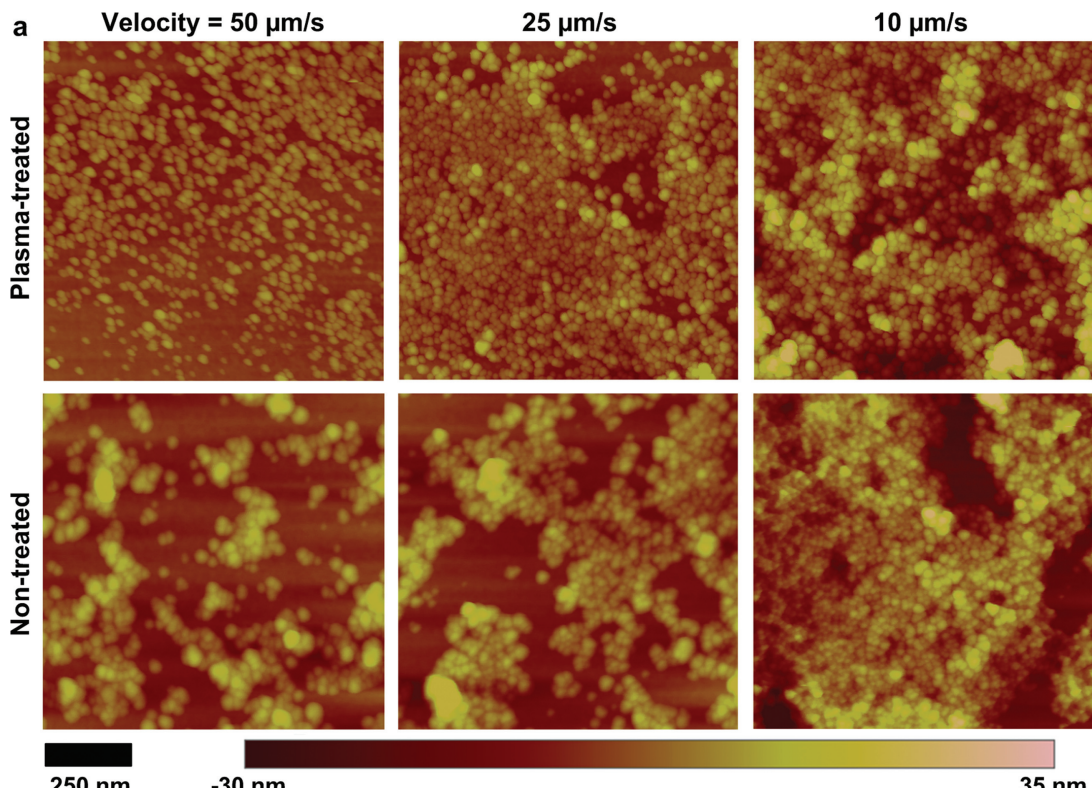

$250 \mathrm{~nm}$

$-30 \mathrm{~nm}$
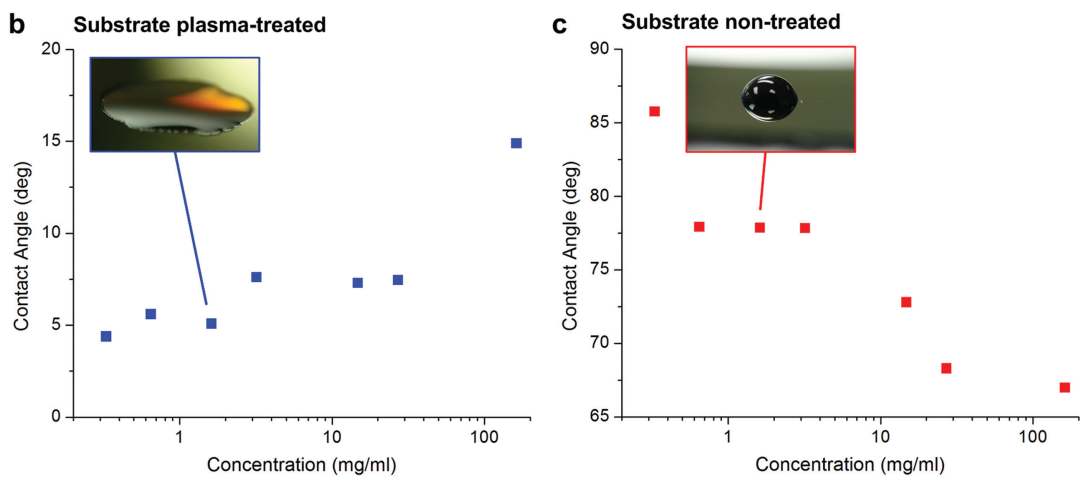

Figure 3. Nanoparticle arrays assembled by blade-casting at various conditions. (a) AFM images of $\mathrm{Al}_{2} \mathrm{O}_{3}$-coated $\mathrm{Si}$ wafer substrates after blade-casting of $3.20 \mathrm{mg} \mathrm{mL}^{-1}$ ferrofluid solution, for plasma-treated substrates (top) and non-treated substrates (bottom), at speeds of 50,25 , and $10 \mu \mathrm{m} \mathrm{s}^{-1}$. Static contact angles between ferrofluid solution and (b) plasmatreated and (c) non-treated substrates.

that the existence of closely spaced yet isolated particles on the plasma-treated substrates resulted in little agglomeration upon annealing in $\mathrm{H}_{2} / \mathrm{He}$ prior to $\mathrm{CNT}$ growth (Supporting Information, Figure S4, Figure S5). Moreover, we found that the density of nanoparticles was directly related to the morphology of carbon nanostructures that are grown on the substrate using a standard atmospheric pressure thermal CVD process. ${ }^{[41]}$

Analysis of the CVD results identified a 'phase diagram' (Figure 4a) with three distinct morphologies of carbon nanostructures: sparse, tangled CNTs; vertically aligned CNTs ('forests'); and high-density carbon 'flakes'. SEM images of these morphologies are shown in Figure $4 b-d$, respectively. We expect that an abrupt transition occurs between tangled and aligned CNTs, as the particle density passes a critical threshold whereby the CNTs are close enough to self-organize into a cooperatively aligned array. ${ }^{[42]}$ When a tangled CNT layer was observed, the corresponding catalyst particle arrays had been fabricated with an insufficient fluid concentration or an excessive blade velocity to form a uniform monolayer, as predicted by Equation 2. As shown in Figure $4 \mathrm{a}$, reduction of the blade velocity at equivalent concentration resulted in growth of a CNT forest. CNT forests were also observed on substrates blade-casted at conditions that we found resulted in multiple layers of nanoparticles.

At even greater particle concentrations in the fluid, the CVD process created the 'flake' morphology, where the carbon is deposited on the catalyst particles in onion-like layers, and these carbon-coated particles form a rigid plate-like microstructure. The carbon is deposited in spherical layers over the catalyst particles, which when combined with the neighboring particles, forms contiguous curled sheets. ${ }^{[43-45]}$ Very few CNTs are formed in this regime, but both TEM imaging (Figure 4d) and Raman spectroscopy (Supporting Information, Figure S6) of the samples indicate that the carbon deposited around the catalyst particles is graphitic. The thick multilayer of iron oxide nanoparticles that are deposited in this regime (verified both by optical color shifts and AFM analysis) yields a large number of particles that are not in contact with the $\mathrm{Al}_{2} \mathrm{O}_{3}$ substrate.

Focusing within the processing regime that leads to CNT forests, we then investigated how the density of the CNT forest could be controlled by the blade-casting process parameters. The volumetric density of each CNT forest was calculated using the measured mass, top-view area, and average height of the forest. We found (Figure 5) that the CNT forest density was controllably influenced by the velocity of blade casting and the concentration of the nanoparticle fluid. Notably, we find that blade-casted catalyst arrays result in CNT forests with densities at least 3-fold higher than those grown from comparable standard thin film dewetting, which typically gives forest densities of 15 to $30 \mu \mathrm{g} \mathrm{mm}^{-3}$ for the same CVD conditions. ${ }^{[19]}$ Spanning our results, blade-casting uniquely enabled tailoring of the CNT forest density from $\sim 1 \mu \mathrm{g} \mathrm{mm}^{-3}$ to $90 \mu \mathrm{g} \mathrm{mm}^{-3}$ simply by specifying the blade velocity and the concentration of the particle solution.

To correlate the CNT density to the morphology and density of the particle layer, we quantified the particle densities from the AFM images of the blade-casted substrates before the CNT growth step. The number of particles was determined using a custom code developed in Matlab and modified from our previous work, ${ }^{[14,19]}$ which identifies the locations of particle peaks in the AFM data. These peaks were then used to perform a Voronoi cell analysis to determine the average particle spacing over the sampled area. Each of the samples that had monolayer particle arrays was analyzed, and a sample of the resulting analysis is presented in 


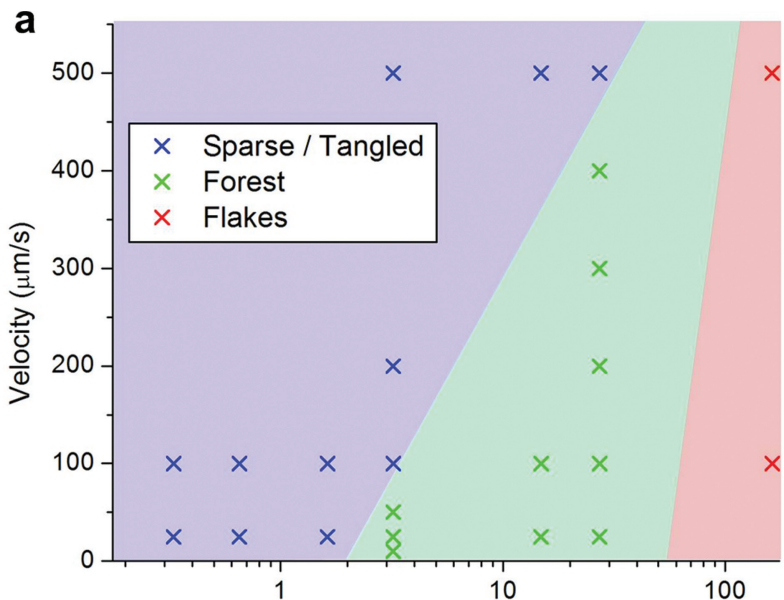

Concentration $(\mathrm{mg} / \mathrm{ml})$
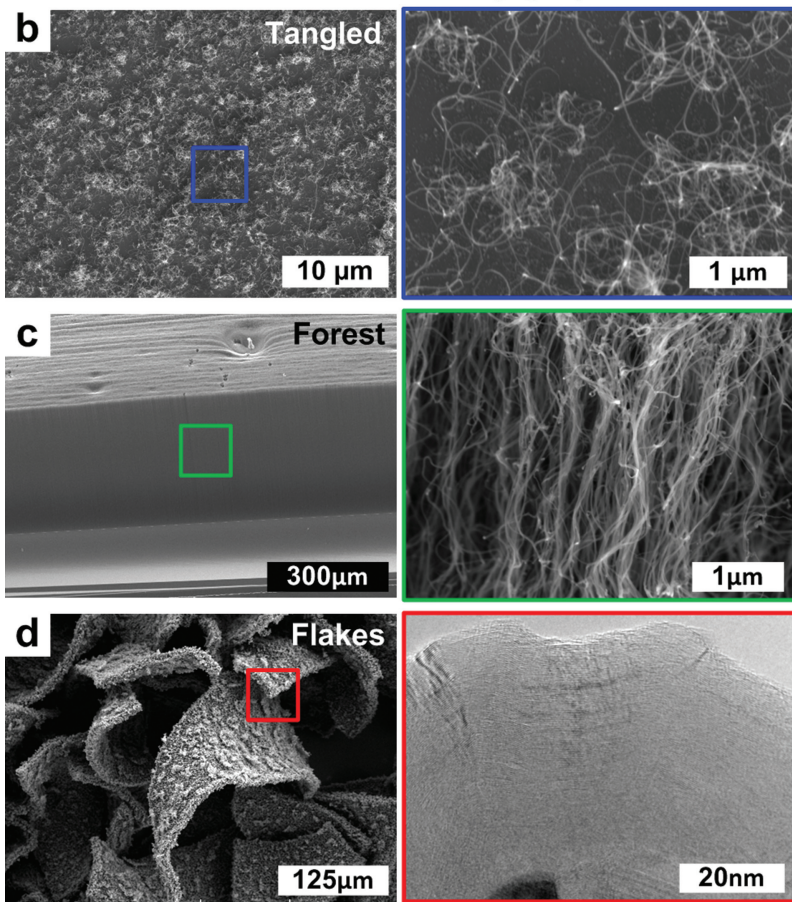

Figure 4. Control of carbon nanostructure morphology by blade-casting parameters, and including process window for CNT forest growth. (a) Relationship between blade velocity, particle solution concentration, and film morphology (tangled, forest, flakes). Data points are from experiments, and lines are drawn to indicate approximate boundaries. (b-d) SEM images for the tangled, forest, and flakes morphologies, respectively. The close-up image in (d) was taken by high-resolution TEM.

Figure 6a. The particle density exhibited an inverse relationship with the blade casting speed, as previously illustrated in Figure 3, which qualitatively matches with the trend in CNT forest density.

\subsection{CNT Diameter and Alignment Analysis}

Next, small angle X-ray scattering (SAXS) was used to nondestructively measure the CNT diameter distribution within the CNT forests. ${ }^{[12,19,46]}$ We found (Figure 7a), that CNT diameter increases slightly with increasing particle number density on the substrate. This trend is consistent for bladecasting conditions that give monolayers and multilayers of catalyst particles. This is due to the presence of additional iron oxide nanoparticles that are in close proximity to each other, which causes the particles to agglomerate during the annealing and reduction phase prior to hydrocarbon exposure. AFM images (Supporting Information, Figure S7) comparing blade-casted substrates before and after annealing show that the magnetite particles coarsen slightly and transition from smooth to faceted texture.

Combining the measurements of CNT diameter and wall thickness by SAXS, the Hermans orientation parameter by SAXS (Supporting Information, Figure S8), and the volumetric density, we then calculated how the number density of CNTs per unit area changes with the process conditions (see Supplemental Information). Comparing the average number of catalyst particles pre-growth, and the average number of CNTs per unit area allowed the particle activation percentage to be calculated (Figure 6b). While the activation percentage for these samples was low, between 0.5 and $6 \%$, there was an increase in catalyst particle activation with an increase in particle packing density, and subsequent VACNT forest volumetric density. Therefore, the tunability of CNT number density is both a direct effect of the particle number density from blade-casting as well as a cooperative increase in the activation percentage (i.e., the proportion of particles that grows CNTs) as shown in Figure 6b. This correlation between the particle areal density and the catalytic activation of particles for CNT growth, suggests a cooperative or autocatalytic nature of CNT growth from catalyst nanoparticles in close proximity. ${ }^{[19,47]}$

It is generally accepted that high-yield activation of catalyst particles for CNT growth on a substrate such as silicon requires an oxide support layer such as $\mathrm{Al}_{2} \mathrm{O}_{3} \cdot{ }^{[48,49]}$ This is illustrated by the lack of VACNT growth on a silicon substrate without $\mathrm{Al}_{2} \mathrm{O}_{3}$, which was prepared identically to a substrate with $\mathrm{Al}_{2} \mathrm{O}_{3}$ that produced a forest. Thus we expected that the significant majority of CNTs grow from particles that are in contact with the $\mathrm{Al}_{2} \mathrm{O}_{3}$ support layer. Accepting this hypothesis suggests that the additional layers of iron oxide nanoparticles influence the activity of the particles in contact with the substrate, such as by increasing the local decomposition of the hydrocarbon source. However, these 'extra' particles do not directly grow CNTs.

\subsection{CNT Diameter and Substrate Engineering}

Another key attribute of the blade-casting process is that the CNT diameter can be specified a priori by the diameter of nanoparticles in the blade-casting solution. To further demonstrate this point, additional experiments were performed using iron oxide nanoparticle solutions (COOH-functionalized, Ocean NanoTech) with nominal diameters of 10, 20 and $30 \mathrm{~nm}$ particles, each having a reported size tolerance of $\pm 2.5 \mathrm{~nm}$. Because the effective particle diameter in solution is influenced by the surface functionalization, we performed 

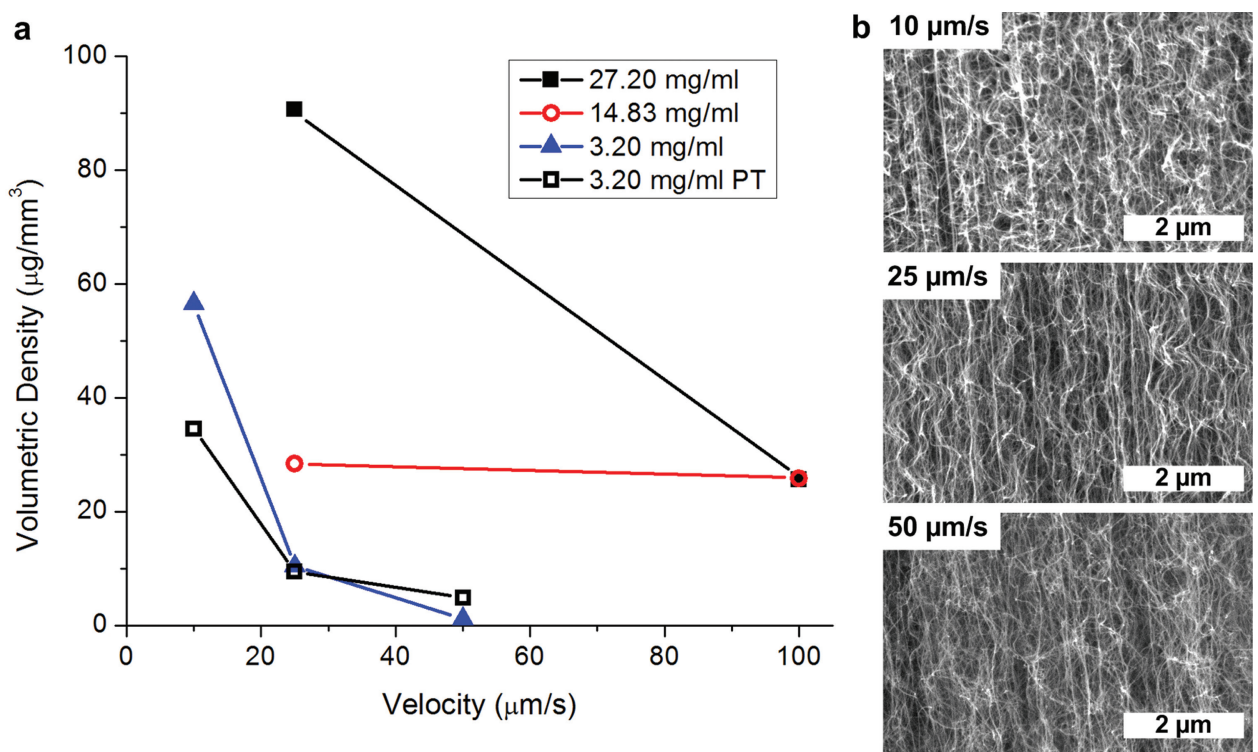

Figure 5. Wide-range control of CNT forest density by blade casting velocity and ferrofluid concentration. (a) Relationship between CNT forest volumetric density and process conditions. (b) SEM images of CNT forest sidewalls grown corresponding to $3.20 \mathrm{mg} \mathrm{mL}^{-1}$ ferrofluid solution at 10 , 25 , and $50 \mu \mathrm{m} \mathrm{s}^{-1}$ (top to bottom).
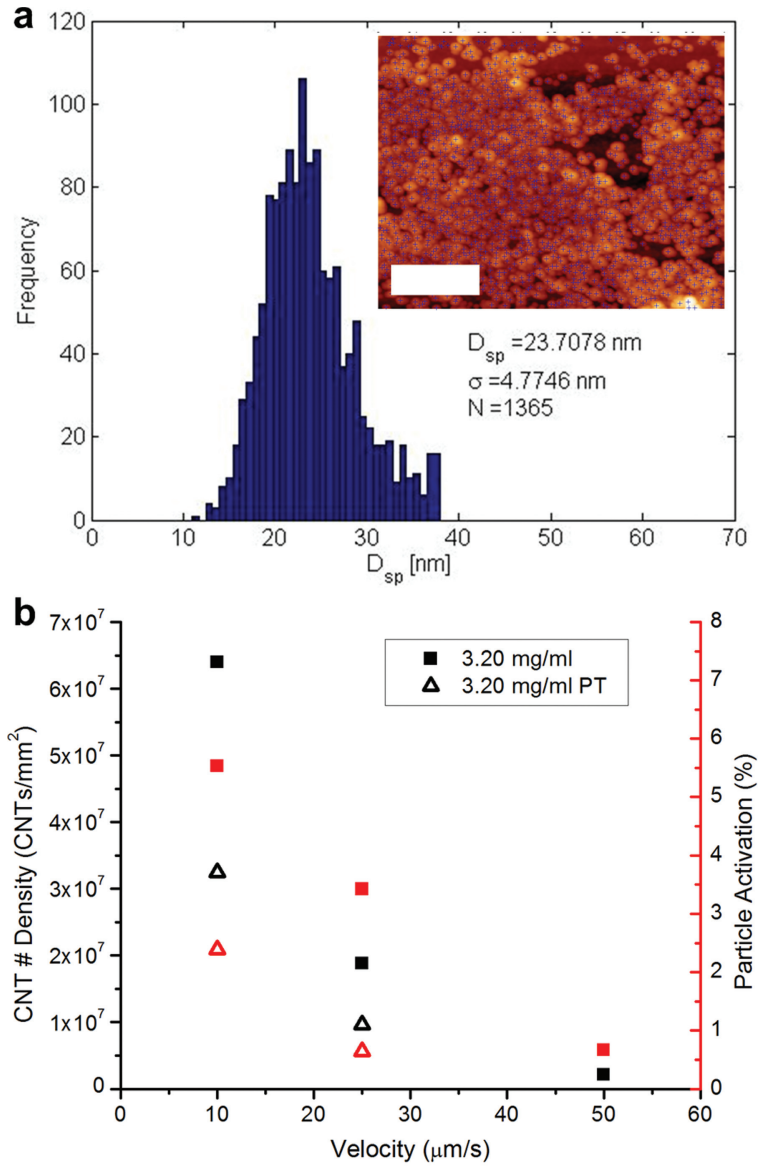

Figure 6. Quantitative analysis of particle density in blade-casted arrays on $\mathrm{Al}_{2} \mathrm{O}_{3}$ substrates. (a) Distribution of inter-particle spacing $\left(D_{s p}\right)$ based on the Voronoi triangulation analysis of a plasma-treated substrate with $3.20 \mathrm{mg} \mathrm{mL}^{-1}$ ferrofluid solution blade casted at $25 \mu \mathrm{m} \mathrm{s}^{-1}$ (inset: AFM image used from this analysis with an area of $1 \times 1 \mu \mathrm{m}$, and a $250 \mathrm{~nm}$ scalebar) and (b) CNT areal density and particle activation measurements for plasma-treated and non-treated substrates. a

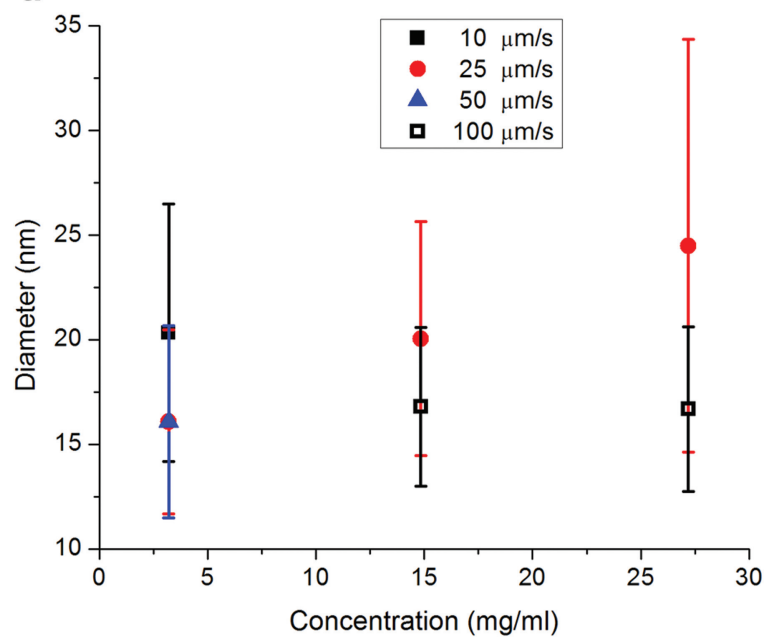

b

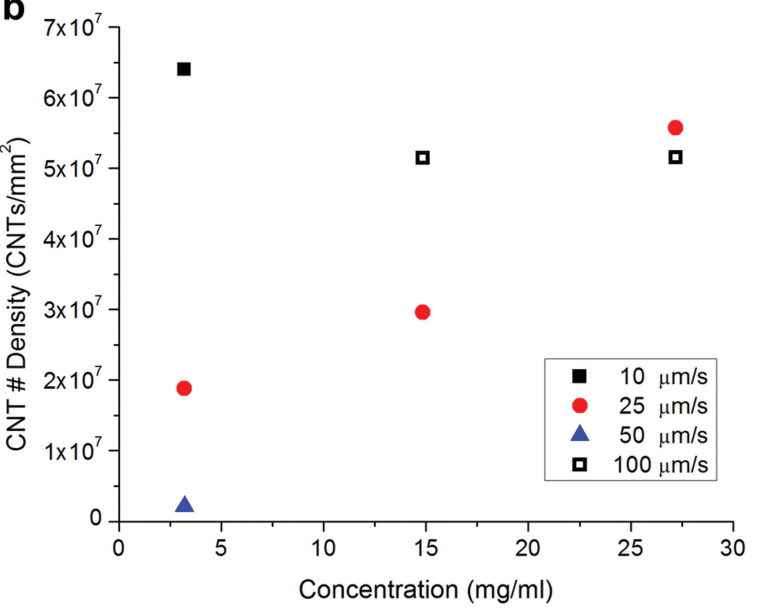

Figure 7. Relationships between (a) CNT diameter and ferrofluid concentration, and (b) CNT number density and ferrofluid concentration, for process conditions giving vertically aligned forests. 

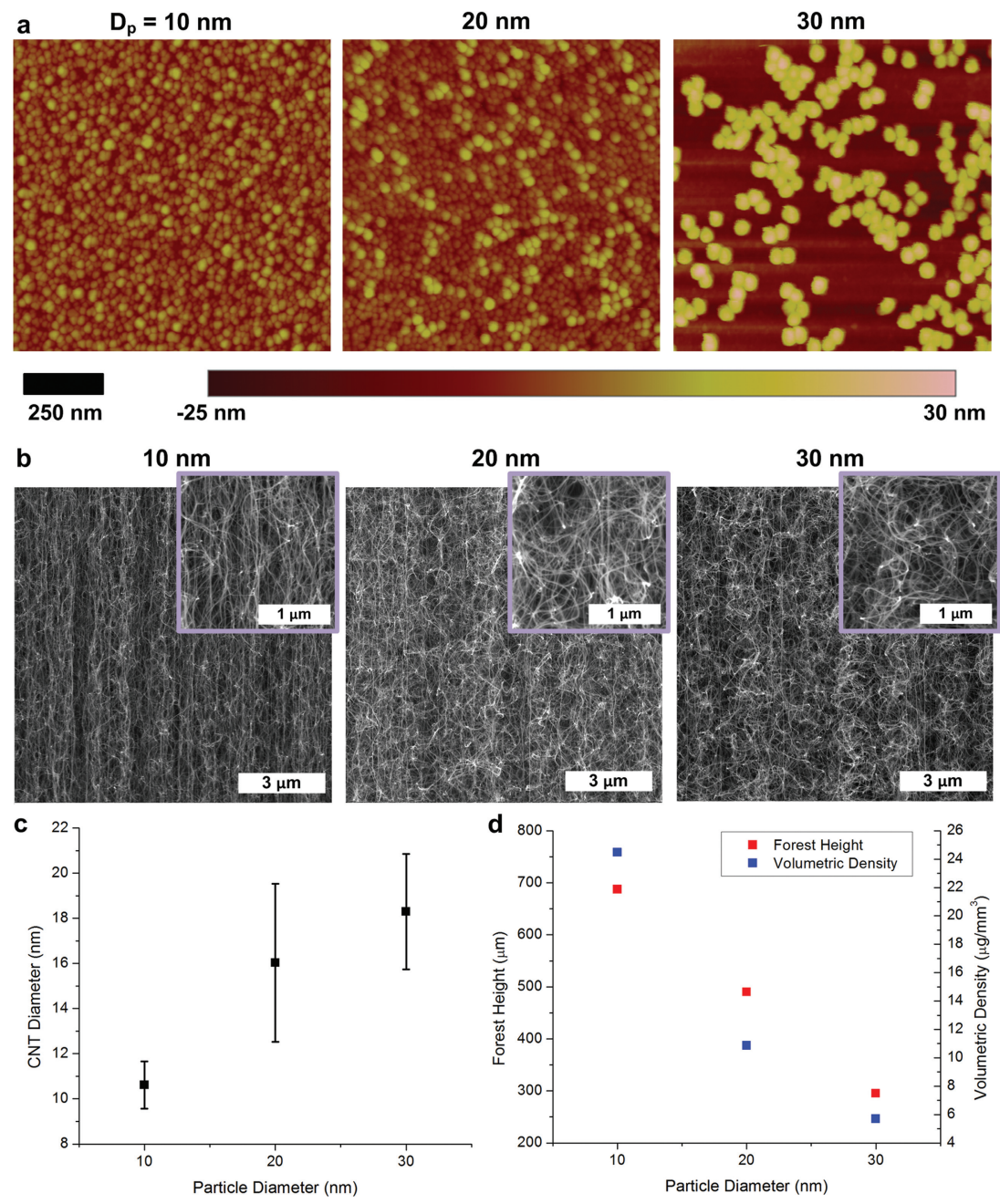

Figure 8. Engineering CNT diameter within forests by specification of the particle diameter in the blade-casting solution. (a) AFM images of $3.20 \mathrm{mg} \mathrm{mL}^{-1}$ ferrofluid solutions with particle diameters of 10, 20 and $30 \mathrm{~nm}$ (left to right) blade casted at $25 \mu \mathrm{m} \mathrm{s}^{-1}$, (b) SEM images of CNT forest sidewalls grown from the three ferrofluid depositions in a), (c) Average CNT diameters for the three CNT forests in (b), and (d) corresponding CNT forest height and volumetric density.

DLS on each as-received solution (Supporting Information, Figure S9).

Based on our findings in the previous sections, solutions with $3.20 \mathrm{mg} \mathrm{mL}^{-1}$ concentration were blade-casted onto plasma-treated substrates at $v_{r}=25 \mu \mathrm{m} \mathrm{s}^{-1}$. As shown in Figure 8a, under these same blade-casting conditions, we obtained a multi-layer deposition of the $10 \mathrm{~nm}$ particles, a near-monolayer of the $20 \mathrm{~nm}$ particles, and a sparse array of the $30 \mathrm{~nm}$ particles. Because the blade casting parameters were held constant, and the same solution concentration was used with each fluid, blade casting of larger diameter particle solutions resulted in fewer particles per unit area, as predicted by Equation 1.

CNT forests were obtained on all samples; however, forests grown from successively larger particles were less dense and less well-aligned (Figure 8b), due to the larger spacing between the catalyst particles on the substrate. A quantitative SEM analysis of the grown forests is shown in Figure $8 \mathrm{c}, \mathrm{d}$, and not only is the trend in VACNT array height and density apparent, but the correlation of CNT diameter to catalyst particle diameter is also very clear. We see that for the three different particle solutions of 10,20 and $30 \mathrm{~nm}$ particles $(14.6 \pm 6.2 \mathrm{~nm}$, $26.3 \pm 8.5 \mathrm{~nm}, 37.2 \pm 11.5 \mathrm{~nm}$ respectively from DLS data), the CNTs grown from them have average diameters of $10.6 \pm 1.0 \mathrm{~nm}, 16.03 \pm 3.5 \mathrm{~nm}, 18.3 \pm 2.6 \mathrm{~nm}$, respectively.

However, while there is a direct correlation between particle size and CNT diameter, the correlation is sub-linear. This finding suggests that the CVD conditions (e.g., feedstock mixture, pressure, temperature) are most favorable for a certain range particle diameters, and that tuning of the CVD conditions would shift the size range of active catalyst particles. Additionally, due to the increasing spacing and decreasing number density with increasing particle diameter in the fabricated arrays, an inverse relationship between particle diameter and forest height, as well as particle diameter and forest density, was observed. Although the particle densities shown here are low due to the parameters chosen, we are confident that optimization of the blade casting parameters could give highly dense arrays of particles of any relevant size. To illustrate this, nearmonolayer arrays of the $30 \mathrm{~nm}$ particles were created by blade-casting the $30 \mathrm{~nm}$ particle solution both at a slower speed, and at a higher concentration (Supporting Information, Figure S10).

Looking ahead, there is strong opportunity to use blade-casting in concert with roll-to-roll processing of flexible substrates. Hence, it is also attractive to grow CNTs on metal foils that are flexible and less expensive than silicon wafers. As an initial demonstration, using the same ferrofluid as earlier in the paper, a $3.20 \mathrm{mg} \mathrm{ml}^{-1}$ solution was blade-casted onto a $\mathrm{Cu}$ foil (first coated with $10 \mathrm{~nm} \mathrm{Al}_{2} \mathrm{O}_{3}$ ) at $25 \mu \mathrm{m} \mathrm{s}^{-1}$. Subsequent growth on the sample resulted in a $\mathrm{CNT}$ forest that visibly covered the area of the $\mathrm{Cu}$ foil that was coated with iron oxide nanoparticles (Figure 9). SEM analysis of the sample showed good alignment of the CNTs at the sidewall of the forest, but due to both the native topography of the substrate and the blade casting conditions, the forest exhibited a high density of micro-cracks. CNT forests on metal foils are particularly desirable for supercapacitor electrodes and thermal interfaces, ${ }^{[50-53]}$ and optimization of this process to a continuous-feed apparatus is ongoing and will be reported in the future. Based on previous work we anticipate that the adhesion can be modulated for specific applications by post-growth treatment, such as cooling the substrate in a hydrocarbon or hydrogen atmosphere. ${ }^{[54]}$ The CNT forests created in this study generally have weak 

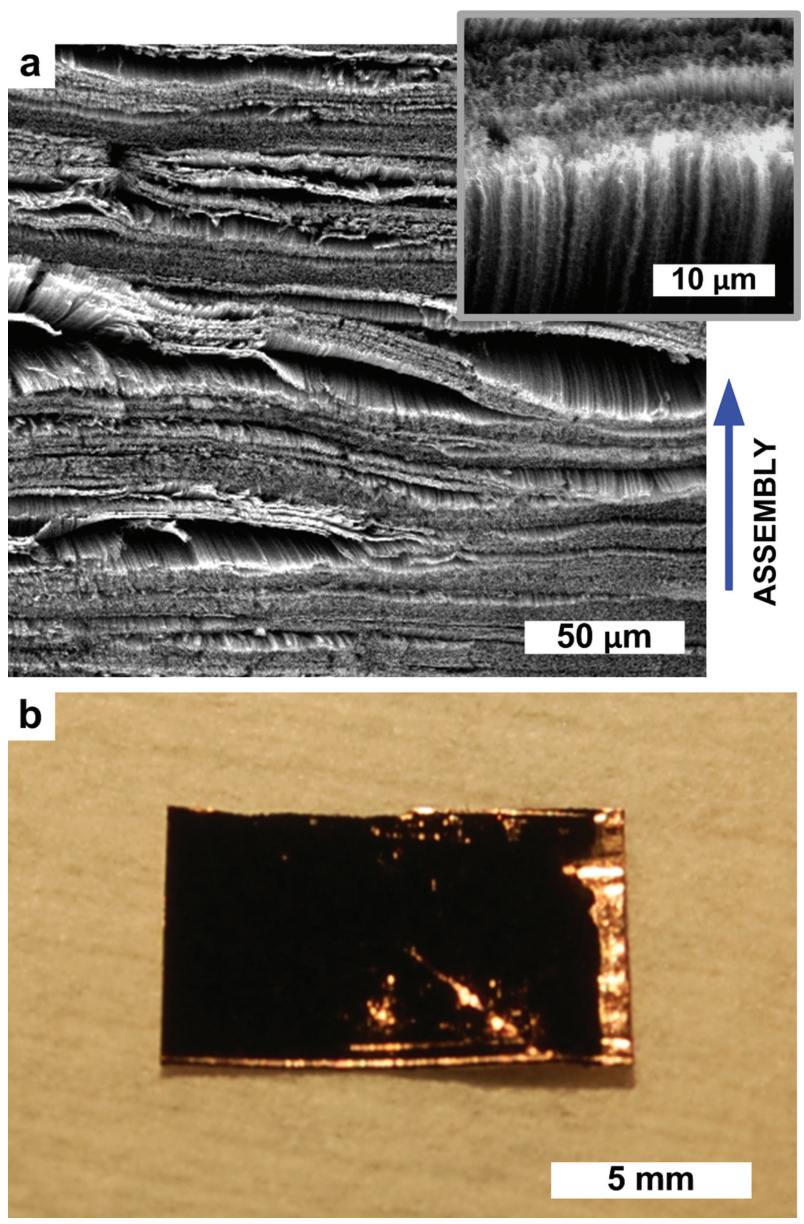

Figure 9. CNT forest grown on Cu foil substrate coated with ferrofluid catalyst array by blade-casting. (a) SEM images of the top and sidewall (inset) of CNT forest grown on Cu foil from a $3.20 \mathrm{mg} \mathrm{mL}^{-1}$ ferrofluid solution blade casted at $25 \mu \mathrm{m} \mathrm{s}^{-1}$ (direction indicated by the arrow). (b) Optical image of the sample from (a). The striped texture of the forest is due to stick-slip of the meniscus, perpendicular to the direction of blade motion.

adhesion to the substrate and can be easily removed using a razor blade.

Last, we comment on the viability of the blade-casting process to obtain truly dense monolayers of nanoparticles. Although we showed that the arrangement of catalyst nanoparticles can be controlled from a sparse sub-monolayer to stacked multi-layers, we did not obtain closely-packed (HCP) monolayers in any experiments. To enable this in the future, it will be most important to use more monodisperse nanoparticle solutions, as are typically used in assembly of 'superlattice' monolayers for other applications. ${ }^{[55-57]}$ Also, to make the process repeatable, precise control over the ambient conditions during blade-casting (e.g., temperature and relative humidity) will be required. The relative humidity of the surrounding environment impacts not only the evaporation rate of the solution during blade-casting, but also can cause considerable effects on the activity and lifetime of CNT growth catalysts. ${ }^{[58-61]}$

The ability to vary the packing fraction, while maintaining a constant average diameter, enables the user to overcome the limits established by dewetting of a thin film, which couples the film thickness, particle diameter, and packing fraction. For this analysis, we denote the packing fraction of particles on the substrate as

$f=N_{p} A_{p}$

where $N_{P}$ is the number of particles per unit substrate area and $A_{P}$ is the projected area of a single particle on the substrate. Conservation of the volume of material deposited as a thin film establishes an inverse relationship between particle diameter and packing fraction after dewetting, ${ }^{[62]}$ as shown in Figure 10. This family of curves assumes the particles are hemispherical in shape.

For a $1 \mathrm{~nm}$ film thickness (which is typical for CNT film growth), with $15 \mathrm{~nm}$ diameter particles, the packing fraction is near $20 \%$, and in order to obtain a larger packing fraction, the catalyst particles would have to be smaller. However, due to the constraint established by minimization of Gibbs free energy, the dewetting of a thin film is limited to a maximum packing fraction of $50 \%$, and thus cannot reach a closely-packed organization. Moreover, because of the complex thermomechanical processes that govern dewetting and stabilization of nanoparticles, it is difficult to independently control the particle diameter and packing fraction, even if the initial film thickness can be very accurately specified. The polydispersity of particle size that is introduced by real attributes of thin film dewetting (e.g., growth of holes, surface roughness), and the propensity for nearby particles to coarsen, further limits the packing fraction to a value below the ideal maximum. Compounded with the typically low yield of CNTs from catalyst particles, this often leads to CNT forests with low packing density and therefore limited properties. In contrast, while the ultimate closely-packed CNT array was

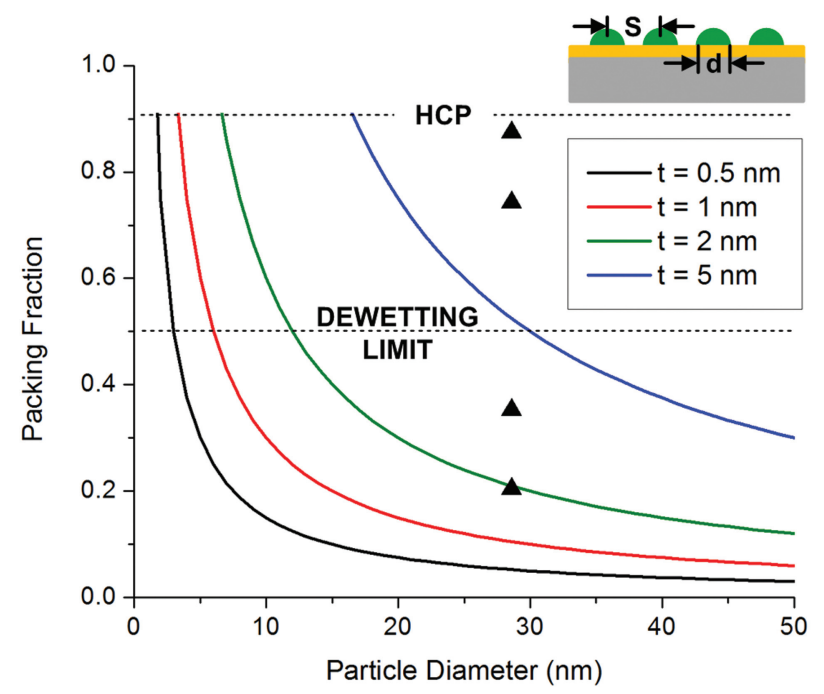

Figure 10. Comparison of nanoparticle array characteristics that can be achieved by thin film dewetting compared to convective assembly. Relationships between particle packing fraction and diameter, for ideal dewetting of films of different thickness are represented by the various curves, while the data points represent several packing fractions achieved from the convective assembly of a $3.20 \mathrm{mg} \mathrm{mL}^{-1}$ ferrofluid (each of which resulted in a CNT forest). 
not achieved in this work, the monolayer arrays made by blade-casting have packing fractions ranging from less than $10 \%$ to approximately $87 \%$ with the same average particle diameter of $28.6 \mathrm{~nm}$.

However, because it is challenging to achieve monodisperse nanoparticles with $<5 \mathrm{~nm}$ diameter, thin films may remain advantageous in depositing ultrathin catalyst layers (i.e., $<1 \mathrm{~nm}$ films), which can generate particles small enough to support SWCNT growth, but at limited densities. For thicker films, especially those generating $>10 \mathrm{~nm}$ particles, the versatility of our approach is clear because it is not possible with any single film thickness to achieve such high packing fractions of catalyst particles and CNTs. Thus, even without meeting the optimal limit of the particle packing, this method for catalyst deposition allows the catalyst particles and thus the VACNT arrays to be tailored to a much higher degree than with standard thin film deposition, while maintaining compatibility with a continuous-feed process.

\section{Conclusion}

We introduced the use of a continuous-feed evaporative selfassembly process (blade-casting) to manufacture arrays of iron oxide nanoparticles, and its application to synthesis of CNT films with controlled organization, diameter, and density. Importantly, this versatile method enables a priori specification of the particle diameter, and independent control of the packing fraction of the arrays by the solution concentration and substrate velocity. As a result, uniform nanoparticle arrays can be made over large areas, ranging from nonclosely packed monolayers to multilayers. Tuning of these two parameters allows unprecedented control of the diameter and density of CNTs with verticaly aligned forests. At high particle concentration, we achieved CNT forest densities 3 -fold higher that a typical thin-film catalyst, and there exists much opportunity to improve upon these values by increasing the catalyst activation percentage and monodispersity of the catalyst particles. Finally, the roll-to-roll compatibility of this method, along with initial results achieved on flexible $\mathrm{Cu}$ foils, suggest promise for scale-up of CNT forest production at commercially relevant throughput.

\section{Experimental Section}

Substrate Preparation: First, $10 \mathrm{~nm}$ of $\mathrm{Al}_{2} \mathrm{O}_{3}$ was deposited by e-beam evaporation on (100) silicon wafers coated with $300 \mathrm{~nm}$ of thermally grown $\mathrm{SiO}_{2}$. The wafers were then manually scribed and fractured into $15 \mathrm{~mm} \times 25 \mathrm{~mm}$ samples. Optionally, the wafer pieces were plasma treated (Harrick Plasma PDC-32G) in air at 200 mTorr, at $6.8 \mathrm{~W}$, for 5 minutes. The samples were then immediately removed from the plasma chamber and loaded onto the blade-casting machine for particle deposition.

Blade-Casting: The custom-built blade-casting apparatus controls the substrate motion using a Thorlabs PT1/M-Z8 single axis translation stage with a Z825B DC servo and TDC001 T-Cube DC motor controller. The blade, a $0.13 \mathrm{~mm}$ thick glass cover slide, is mounted rigidly to a vertical support and oriented $20^{\circ}$ from the vertical axis. The blade edge is first brought into contact with the substrate using a vertical micrometer stage (Thorlabs PT1/M), and $6 \mu \mathrm{L}$ of the ferrofluid solution (Ferrotec MSGW11 and Oceantech SHP-10, SHP-20 and SHP-30) is placed at the interface between the blade and the substrate, using a handheld micropipette. The solution spreads along the interface, forming a static meniscus between the two surfaces over the $15 \mathrm{~mm}$ width of the substrate. After the meniscus is formed, the substrate motion is activated at a constant velocity. During this time, the dynamic contact angle and meniscus shape were observed to determine if the meniscus was pinning sufficiently to the substrate to enable particle deposition. Once the blade reaches the end of the substrate, the remaining ferrofluid is removed from the substrate using the edge of a clean Kimwipe, which gently wicks the fluid away from the deposited area. Finally, the sample is placed on a hotplate at $75^{\circ} \mathrm{C}$ for $30 \mathrm{~min}$ to ensure the complete evaporation of water. All blade casting experiments were conducted with local ambient temperature and relative humidity values at $22.0 \pm 0.3{ }^{\circ} \mathrm{C}$ and $31.3 \pm 4.9 \%$ respectively.

CNT Growth: CNT growth was performed in a Lindberg Blue $M$ horizontal tube furnace (22 mm ID, $300 \mathrm{~mm}$ heated length) at atmospheric pressure, with flows of $100 / 100 / 400 \mathrm{sccm} \mathrm{C}_{2} \mathrm{H}_{4}$ l $\mathrm{H}_{2} / \mathrm{He}$, at $775{ }^{\circ} \mathrm{C}$ for $20 \mathrm{~min}$, preceded by an annealing step at $775{ }^{\circ} \mathrm{C}$ for 10 min with flows of $400 / 100 \mathrm{sccm} \mathrm{H} \mathrm{H}_{2} / \mathrm{He}$. The CNTs were rapidly cooled in the growth atmosphere before purging the CVD chamber with He when the thermocouple reading drops below $200{ }^{\circ} \mathrm{C}$. An identical recipe was used for all experiments reported in this paper, and this recipe yielded the different morphologies discussed in the text.

Characterization: AFM imaging was performed in tapping mode in a Veeco Dimension Icon. Mass measurements of the substrates were collected after nanoparticle deposition and after CNT growth, using an Ohaus Discovery microbalance, and the differential represented the mass of CNTs grown on the substrate. The areal coverage of each VACNT array was calculated using contrast pixel counting in Adobe Photoshop CS6, using images taken by a Nikon D40 camera. SEM imaging was performed using a FEI Nova Nanolab, and CNT diameters and forest heights were measured directly from SEM images at different magnifications. The combination of the mass, area and height measurements was used to calculate the areal and volumetric densities of the VACNT arrays. TEM images of the 'flake' structures were collected using a JEOL 3011 HREM.

For X-ray scattering measurements, the CNT sample was placed on a motorized stage in the beam path of the $\mathrm{G} 1$ beamline at Cornell High Energy Synchrotron Source (CHESS). A beam energy of $10 \pm$ $0.1 \mathrm{keV}$ (wavelength $\approx 0.13 \mathrm{~nm}$ ) is selected with synthetic multilayer optics (W/B4C, 27.1 $\mathrm{d}$ d-spacing), and the beam was focused down to $\approx 20 \mu \mathrm{m}$ using mechanical slits upstream. The beam size is accurately measured by scanning the beam over a pinhole slit mounted on a motorized stage while measuring the beam intensity. A standard sample of silver behenate powder $\left(d_{001}=58.380 \AA\right)$ is used to calibrate the pixel-to-q ratio. Linescans from the 2D SAXS patterns are fitted using a mathematical model for lognormally distributed hollow cylinders. These scans are obtained by integration of intensities within $\pm 10^{\circ}$ from the reference direction ( $x$-axis) of the inverse space parameter $\mathrm{q}$ (chosen to be the direction of maximum intensity). The fitting code used an iterative approach in searching for the best fit within a user defined fitting range. By including the low q part of the data, a good fit was achieved that selects a 
probability density function (PDF) for diameter distribution as well as for the ratio $c=I D / O D$, where ID is the inner diameter of the multi-wall CNT and OD is the outer diameter. ${ }^{[12,46]}$

\section{Supporting Information}

Supporting Information is available from the Wiley Online Library or from the author.

\section{Acknowledgements}

We thank Eric R. Meshot for his contributions to the AFM particle analysis code, and Arthur Woll for assistance with X-ray scattering data collection. This work was supported by the Nanomanufacturing program of the National Science Foundation (CMMI-0927634); the Scalable Nanomanufacturing Program of the National Science Foundation (DMR-1120187); the Office of Naval Research (N0001411M0217) via a subcontract to the from Absolute Nano, LLC to the University of Michigan; and the DoD, Air Force Office of Scientific Research, National Defense Science and Engineering Graduate (NDSEG) Fellowship, 32 CFR 168 a awarded to E.S.P. X-ray scattering was performed in the G1 beamline at the Cornell HighEnergy Synchrotron Source (CHESS), which is supported by the NSF and the National Institutes of Health under Grant DMR-0225180. Electron microscopy and AFM were performed at the University of Michigan Electron Microbeam Analysis Library (EMAL), and microfabrication was performed at the Lurie Nanofabrication Facility (LNF) which is a member of the National Nanotechnology Infrastructure Network. A.J.H. is a partner of Absolute Nano, LLC.

[1] B. J. Hinds, N. Chopra, T. Rantell, R. Andrews, V. Gavalas, L. G. Bachas, Science 2004, 303, 62-65.

[2] A. Kalra, S. Garde, G. Hummer, Proc. Natl. Acad. Sci. USA 2003, 100, 10175-10180.

[3] L. Sun, R. M. Crooks, J. Am: Chem. Soc. 2000, 122, $12340-12345$

[4] E. J. Garcia, B. L. Wardle, John Hart, Composites Part A 2008, 39, 1065-1070.

[5] T. Tong, Y. Zhao, L. Delzeit, A. Kashani, M. Meyyappan, A. Majumdar, IEEE Trans. Components Packaging Technol. 2007, $30,92-100$

[6] F. Javier del Campo, J. García-Céspedes, F. Xavier Muñoz, E. Bertrán, Electrochem. Commun. 2008, 10, 1242-1245.

[7] E. Garcia, B. Wardle, A. J. Hart, N. Yamamoto, Composites Sci. Technol. 2008, 68, 2034-2041.

[8] S. Fan, W. Liang, H. Dang, N. Franklin, T. Tombler, M. Chapline, H. Dai, Phys. E 2000, 8, 179-183.

[9] S. Fan, M. G. Chapline, N. R. Franklin, T. W. Tombler, A. M. Cassell, H. Dai, Science 1999, 283, 512-514.

[10] S. Hofmann, R. Sharma, C. Ducati, G. Du, C. Mattevi, C. Cepek, M. Cantoro, S. Pisana, A. Parvez, F. Cervantes-Sodi, A. C. Ferrari, R. Dunin-Borkowski, S. Lizzit, L. Petaccia, A. Goldoni, J. Robertson, Nano Lett. 2007, 7, 602-608.

[11] S. Esconjauregui, M. Fouquet, B. C. Bayer, C. Ducati, R. Smajda, S. Hofmann, J. Robertson, ACS Nano 2010, 4, 7431-7436.
[12] E. R. Meshot, D. L. Plata, S. Tawfick, Y. Zhang, E. A. Verploegen, A. J. Hart, ACS Nano 2009, 3, 2477-2486.

[13] Y. Zhang, Y. Li, W. Kim, D. Wang, H. Dai, Appl. Phys. A: Mater. Sci. Processing 2002, 74, 325-328.

[14] E. R. Meshot, E. Verploegen, M. Bedewy, S. Tawfick, A. R. Woll, K. S. Green, M. Hromalik, L. J. Koerner, H. T. Philipp, M. W. Tate, S. M. Gruner, A. J. Hart, ACS Nano 2012, 6, 5091-5101.

[15] R. D. Bennett, A. J. Hart, R. E. Cohen, Adv. Mater. 2006, 18, 2274-2279.

[16] G. D. Nessim, A. J. Hart, J. S. Kim, D. Acquaviva, J. Oh, C. D. Morgan, M. Seita, J. S. Leib, C. V Thompson, Nano Lett. 2008, 8, 3587-3593.

[17] K. Yoshihara, S. Honda, J.-G. Lee, H. Mori, K. Oura, M. Katayama, Japanese J. Appl. Phys. 2008, 47, 1941-1943.

[18] M. Bedewy, E.R. Meshot, A. J. Hart, Carbon 2012, 50, 5106-5116.

[19] M. Bedewy, E. R. Meshot, M. J. Reinker, A. J. Hart, ACS Nano 2011 5, 8974-8989.

[20] Y. T. Chou, Y. T. Ko, M. F. Yan, J. Am. Ceram. Soc. 1987, 70, 280-282.

[21] H. J. Kim, M. J. M. Krane, K. P. Trumble, K. J. Bowman, J. Am. Ceram. Soc. 2006, 89, 2769-2775.

[22] B. G. Prevo, O. D. Velev, Langmuir 2004, 20, 2099-2107.

[23] L. Malaquin, T. Kraus, H. Schmid, E. Delamarche, H. Wolf, Lang muir 2007, 23, 11513-11521.

[24] P. Kumnorkaew, Y. Ee, N. Tansu, J. F. Gilchrist, Langmuir 2008, 24, 12150-12157.

[25] M. I. Bodnarchuk, M. V Kovalenko, S. Pichler, G. Fritz-Popovski, G. Hesser, W. Heiss, ACS Nano 2010, 4, 423-431.

[26] H. Yang, P. Jiang, Langmuir 2010, 26, 13173-13182.

[27] Y. Tian, B. Yu, X. Li, K. Li, J. Mater. Chem. 2011, 21, 2476-2481.

[28] Y. Zhu, F. Y. Jiang, K. Chen, F. Kang, Z. K. Tang, J. Alloys Compounds 2011, 509, 8549-8553.

[29] C. Y. Wang, J. M. Hong, G. Chen, Y. Zhang, N. Gu, Chinese Chem. Lett. 2010, 21, 179-182.

[30] S. Guo, D. Li, L. Zhang, J. Li, E. Wang, Biomaterials 2009, 30, 1881-1889.

[31] J. Salado, M. Insausti, I. Gil de Muro, L. Lezama, T. Rojo, J. Non-Crystalline Solids 2008, 354, 5207-5209.

[32] W. W. Yu, J. C. Falkner, C. T. Yavuz, V. L. Colvin, Chem. Commun. 2004, 40, 2306-2307.

[33] S. G. Kwon, Y. Piao, J. Park, S. Angappane, Y. Jo, N.-M. Hwang, J.-G. Park, T. Hyeon, J. Am. Chem. Soc. 2007, 129, 12571-12584.

[34] N. T. Alvarez, F. Li, C. L. Pint, J. T. Mayo, E. Z. Fisher, J. M. Tour, V. L. Colvin, R. H. Hauge, Chem. Mater. 2011, 23, 3466-3475.

[35] C.-H. Chang, H. J. In, S. Takahashi, M. Deterre, H. J. Choi, K. W. Gotrik, G. Barbastathis, Nanotechnology 2011, 22, 035301.

[36] G. S. Choi, Y. S. Cho, K. H. Son, D. J. Kim, Microelectron. Engin. 2003, 66, 77-82.

[37] B. G. Prevo, D. M. Kuncicky, O. D. Velev, Colloids Surf. A 2007, 311, 2-10.

[38] B. G. Prevo, J. C. Fuller, O. D. Velev, Chem. Mater. 2005, 17, 28-35.

[39] T. P. Bigioni, X.-M. Lin, T. T. Nguyen, E. I. Corwin, T. A. Witten, H. M. Jaeger, Nat. Mater. 2006, 5, 265-270.

[40] A. S. Dimitrov, K. Nagayama, Langmuir 1996, 12, 1303-1311.

[41] A. J. Hart, A. H. Slocum, J. Phys. Chem. B 2006, 110, 8250-8257.

[42] M. Bedewy, E. R. Meshot, H. Guo, E. A. Verploegen, W. Lu, A. J. Hart, J. Phys. Chem. C 2009, 113, 20576-20582.

[43] T. Cabioc'h, E. Thune, J. P. Riviére, S. Camelio, J. C. Girard, P. Guérin, M. Jaouen, L. Henrard, P. Lambin, J. Appl. Phys. 2002 91, 1560-1567.

[44] C. He, N. Zhao, C. Shi, X. Du, J. Li, L. Cui, J. Alloys Compounds 2006, 425, 329-333.

[45] Z. Abdullaeva, E. Omurzak, C. Iwamoto, H. S. Ganapathy, S. Sulaimankulova, C. Liliang, T. Mashimo, Carbon 2012, 50, 1776-1785. 
[46] B. N. Wang, R. D. Bennett, E. Verploegen, A. J. Hart, R. E. Cohen, J. Phys. Chem. C 2007, 111, 5859-5865.

[47] N. Latorre, E. Romeo, F. Cazana, T. Ubieto, C. Royo, I. Villacampa, A. Monzon, J. Phys. Chem. C 2010, 114, 4773-4782.

[48] P. B. Amama, C. L. Pint, F. Mirri, M. Pasquali, R. H. Hauge, B. Maruyama, Carbon 2012, 50, 2396-2406.

[49] T. D. L. Arcos, F. Vonau, M. G. Garnier, V. Thommen, H.-G. Boyen, P. Oelhafen, Appl. Phys. Lett. 2002, 80, 2383-2385.

[50] L. Gao, A. Peng, Z. Y. Wang, H. Zhang, Z. Shi, Z. Gu, G. Cao, B. Ding, Solid State Commun. 2008, 146, 380-383.

[51] D. N. Futaba, K. Hata, T. Yamada, T. Hiraoka, Y. Hayamizu, Y. Kakudate, O. Tanaike, H. Hatori, M. Yumura, S. lijima, Nat. Mater. 2006, 5, 987-994.

[52] H. Wang, J. Feng, X. Hu, K. M. Ng, J. Phys. Chem. C 2007, 111, 12617-12624.

[53] W. Lin, R. Zhang, K.-S. Moon, C. Wong, IEEE Trans. Adv. Packaging 2010, 33, 370-376.

[54] C. L. Pint, Y.-Q. Xu, M. Pasquali, R. H. Hauge, ACS Nano 2008, 2, 1871-1878.
[55] M. Retsch, Z. Zhou, S. Rivera, M. Kappl, X. S. Zhao, U. Jonas, Q. Li, Macromol. Chem. Phys. 2009, 210, 230-241.

[56] A. Dong, X. Ye, J. Chen, C. B. Murray, Nano Lett. 2011, 11, 1804-1809.

[57] T. Wen, S. Majetich, ACS Nano 2011, 5, 8868-8876.

[58] D. Futaba, K. Hata, T. Yamada, K. Mizuno, M. Yumura, S. lijima, Phys. Rev. Lett. 2005, 95, 056104.

[59] F. L. Deepak, A. Govindaraj, C. N. R. Rao, J. Chem. Sci. 2006, 118, 9-14.

[60] K. Hata, D. N. Futaba, K. Mizuno, T. Namai, M. Yumura, S. Iijima, Science 2004, 306, 1362-1364.

[61] A. Cao, X. Zhang, C. Xu, J. Liang, D. Wu, B. Wei, J. Mater. Res. 2011, 16, 3107-3110.

[62] J. Robertson, G. Zhong, C. S. Esconjauregui, B. C. Bayer, C. Zhang, M. Fouquet, S. Hofmann, Japanese J. Appl. Phys. 2012, 51, $01 \mathrm{AH} 01$.

Received: November 19, 2012

Revised: December 20, 2012

Published online: February 18, 2013 